\title{
Stimulus and response priming in rapid serial visual presentation: Evidence for a dissociation
}

\author{
ELKAN G. AKYÜREK \\ Leiden University, Leiden, The Netherlands \\ and University of Reading, Reading, England \\ AND \\ BERNHARD HOMMEL \\ Leiden University, Leiden, The Netherlands
}

\begin{abstract}
Two experiments investigated the capacity demands of stimulus and response priming in a rapid serial visual presentation (RSVP) task. Three targets were presented in a stream of visual symbols: The first two (T1 and T2) required an unspeeded manual response at the end of the trial, but the third (T3) called for an immediate, speeded manual response. T2 and T3 either were identical (fully compatible), required the same response (response compatible), or required different responses (incompatible). Priming in the fully compatible condition depended strongly on successful identification of the priming stimulus, whereas response-based priming was observed regardless of whether the prime could be reported or not. These findings suggest that stimulus coding and response coding are automatic processes, unaffected by attentional capacity constraints, followed by capacity-limited stimulus consolidation and response selection. Moreover, even though response codes are activated automatically upon stimulus processing, both types of codes act, and affect behavior, independently.
\end{abstract}

One of the most robust demonstrations of the limitations underlying human attention is the so-called attentional blink (AB). The AB occurs when people monitor a stream of stimuli for multiple targets in a rapid serial visual presentation (RSVP) task. Whereas the first target (T1) can normally be reported with high accuracy, a second target (T2) is often missed if it occurs too soon (around 100-600 msec) after the first (Broadbent \& Broadbent, 1987; Raymond, Shapiro, \& Arnell, 1992; Schneider \& Shiffrin, 1977). This suggests that processing a target for later report creates a transient attentional bottleneck that prevents the complete processing of other targets. However, this does not mean that later targets are not processed at all. On the contrary, analyses of the side effects and aftereffects of unreported targets strongly suggest that their identity and meaning are successfully registered by the cognitive system.

One piece of evidence comes from the electrophysiological study of Luck, Vogel, and Shapiro (1996), who found a reliable T2-induced N400 for trials in which T2 could not be reported (see also Vogel, Luck, \& Shapiro, 1998). Given that the N400 component is commonly taken to indicate semantic processing, this suggests that $\mathrm{T} 2$ was identified at some level. Further evidence comes from Shapiro, Driver, Ward, and Sorensen (1997), who investigated whether a missed T2 would be able to prime a subsequent T3. In their first experiment, which employed a design similar to the one used in the present experiments, they found substantial repetition priming or, more precisely, repetition blindness. In their second experiment, they used words that were or were not semantically related, and found that related words facilitated performance, even if the priming word was missed. The latter effect was replicated in a similar task by Martens, Wolters, and van Raamsdonk (2002).

These findings demonstrate that a great deal of processing, such as the identification of a stimulus and the activation of stimulus-related episodic and semantic traces, takes place in the absence of conscious awareness. In the present study, we asked whether processing goes even further-in particular, whether it extends to the actions related to a missed stimulus. If so, this would indicate that translating a stimulus into a response does not share capacity with the resources underlying the $\mathrm{AB}$. There are reasons to assume that this might be the case. In several single-task studies, it has been found that unnoticed and nonreportable stimuli can activate arbitrary responses assigned to them (Eimer \& Schlaghecken, 1998; Leuthold \& Kopp, 1998; Neumann \& Klotz, 1994). Along the same lines, dual-task studies have shown that stimulus-response translation is not prevented in the presence of an attentional bottleneck: Responses for a secondary task are activated before primary-task processing is completed (Hommel, 1998; Hommel \& Eglau, 2002; Logan \& Schulkind, 2000). On the other hand, Jolicœur and colleagues (e.g., Jolicœur \& Dell'Acqua, 1998; for an overview and theoretical sum- 
mary, see Jolicœur, Tombu, Oriet, \& Stevanovski, 2002) have repeatedly found that the $\mathrm{AB}$ interacts with response selection (as opposed to response activation) processes. The extent to which response-related processing is independent of the $A B$ is thus not clear. The findings reported to date raise the questions of how far nonreportable stimuli can be processed and whether they activate their assigned responses when facing an attentional bottleneck. To investigate these questions, we examined whether a missed $\mathrm{T} 2$ in an $\mathrm{AB}$ task activates its stimulus representation and (even more importantly) its response. In other words, we tested whether and to what degree stimulus and response activation can proceed without attention.

We investigated this issue in two experiments by applying the logic of Shapiro et al. (1997) to response priming. That is, we presented participants with an RSVP stream that contained two targets, $\mathrm{T} 1$ and $\mathrm{T} 2$, for later, unspeeded report and a third target (T3) requiring an immediate, speeded binarychoice response, in order to ensure online response selection for T3 (see Jolicœur, 1998). The relations between the identities of T2 and T3 and between the responses these two targets required were systematically varied. In a fully compatible condition, $\mathrm{T} 2$ and $\mathrm{T} 3$ were identical and, hence, required the same manual response; in a response-compatible condition, the two targets differed but still required the same response; and in an incompatible condition, both the targets and their associated responses were different. These three conditions allow for two comparisons of theoretical interest. First, comparing the response-compatible and the fully compatible conditions provides an estimate of priming that can be attributed to particular stimulus codes, which then enables us to relate our findings to previous studies investigating stimulus priming. Second, comparing the responsecompatible and the incompatible conditions provides an estimate of response priming, which enables us to assess the degree to which priming effects go beyond similar activation. Moreover, deriving these two priming-activation measures made it possible to compare the effects due to stimulus and response activation and to see whether these effects are sensitive to the same manipulations. Of special interest was, first, whether performance on $\mathrm{T} 3$ would be better when the responses to T2 and to T3 matched and, second, whether this response-priming effect would depend on successful report of T2. Furthermore, to test whether the mere activation of a response, without reinforcement by selection, is a less durable cognitive event, in Experiment 2 we manipulated the available time between $\mathrm{T} 2$ and $\mathrm{T} 3$ to investigate response-priming effects over time.

\section{EXPERIMENT 1}

To investigate priming contingent on $\mathrm{T} 2$, the first experiment elaborated on a basic RSVP design, as commonly employed to investigate the $\mathrm{AB}$, by adding a third target, in an experimental design similar to those in the studies of Shapiro et al. (1997) and Loach and Marí-Beffa (2003). The distractors in the stream were letters, and T1 was a digit. T2 and T3 were symbol characters associated through instruction with left or right keypresses. T1 and T2 were reported at leisure at the end of the trial, as is common in RSVP stud- ies, but $\mathrm{T} 3$ required an immediate, speeded response. Of primary interest was the compatibility relation between $\mathrm{T} 2$ and T3 - that is, whether or not they required the same response and whether their visual appearance was identical.

\section{Method}

Participants. Seventy students participated in the experiment in exchange for course credit or monetary compensation. All of them reported having normal (or corrected-to-normal) vision and concentration span.

Apparatus and Stimuli. All stimuli were presented at a resolution of $800 \times 600$ pixels, in 16-bit color, on a 17-in. CRT monitor refreshing at $100 \mathrm{~Hz}$. The experimental program ran on a Pentium III $\mathrm{PC}$ and was programmed in E-Prime 1.1 SP3. The participants were seated individually in small, dimly lit cabins at a viewing distance of about $50 \mathrm{~cm}$ from the monitor. The fixation mark ("+") and all RSVP items were presented centrally in black on a gray background (RGB: $128,128,128)$, with the exception of the first and third targets, which were presented in soft white (RGB: 220, 220, 220) and bright red (RGB: 255, 0,0), respectively. Each item was set in 16-point Times New Roman font. Digits were drawn randomly without replacement from 1-9, with the exclusion of 5. Distractors were drawn from the full alphabet. The symbol characters used for T2 and T3 were "\#," “\%," "\&," and “@,."The stimulus-response mapping was counterbalanced: One half of the participants pressed a left key for the targets "\#" and "\%" and a right key for the targets " \&" and "@," and the other half received the opposite mapping. Left and right responses were given with the $Q$ and $P$ keys on the keyboard, which were relabeled with arrow symbols pointing in their respective directions.

Compatibility between $\mathrm{T} 2$ and $\mathrm{T} 3$ was manipulated as follows: On $50 \%$ of the trials, T2 and T3 were incompatible; that is, the two target symbols and the associated responses were different. On 25\% of the trials, the two target symbols differed but required the same response (response compatible). On the remaining $25 \%$ of the trials, the two symbols and the two responses were the same (fully compatible). The main measure was the identification accuracy for the target stimuli. T3 required a speeded identification response, and accordingly, reaction time (RT) was recorded.

Procedure and Design. Participants initiated each trial by pressing the space bar. Trials started with a delay of $800 \mathrm{msec}$, followed by the presentation of the fixation mark for $200 \mathrm{msec}$. The RSVP ensued, consisting of 20 items with a duration of $70 \mathrm{msec}$ each and an interstimulus interval of $30 \mathrm{msec}$. Participants were instructed to respond to $\mathrm{T} 3$ by pressing the associated key as soon as they detected the target in the stream. The end of the stream was followed by a 1,000-msec blank pause, after which two response input screens were presented for reporting the first two targets. T1 was identified by pressing the corresponding digit key, and $\mathrm{T} 2$ was reported by pressing the associated key on the keyboard (using the same mapping as T3). There was no option to omit a response.

A full experimental session lasted for approximately $1 \mathrm{~h}$ and contained two blocks of 256 randomly ordered trials and 20 practice trials. The design consisted of two within-participants variables: T2 lag and T2-T3 compatibility. Lag was determined by the number of items between T1 and T2. Performance was measured at lag 2 and at lag 8 , lags at which $\mathrm{T} 2$ would be likely to fall within and outside the $\mathrm{AB}$, respectively. T1 position was randomly varied between Stream Positions 7 and 8 to reduce the predictability of target onsets. T3 always followed T2 at a lag of two items; that is, T2-T3 lag was constant. The T2-T3 compatibility variable consisted of three levels: incompatible, response compatible, and fully compatible.

\section{Results and Discussion}

Repeated measures ANOVAs were carried out on (1) accuracy on T1; (2) accuracy on T2, given that the response to T1 was correct (T2|T1); and (3) accuracy as well as RT on T3, given that T2 was correct (T3|T2). For 
the RT analyses, correct as well as incorrect responses were included. The independent variables were T1-T2 lag and T2-T3 compatibility. To safeguard against spurious keypresses, we excluded trials in which no valid response was detected within the interval of 100-1,300 msec after T3 onset. When necessary, the degrees of freedom were Greenhouse-Geisser adjusted in case of a significant sphericity test (rounded to one decimal).

T1. Figure 1 shows $\mathrm{T} 1$ and conditional T2 accuracies over all conditions of the experiment. As expected, no variable reached significance in the $\mathrm{T} 1$ analysis $(F<1$ for lag, $F<2.4$ for compatibility, and $F<1.9$ for their interaction).

T2|T1. Accuracy on T2 was affected by both T1-T2 lag $\left[F(1,69)=76.81, M S_{\mathrm{e}}=.003, p<.001\right]$ and compatibility $\left[F(1.6,111.5)=16.15, M S_{\mathrm{e}}=.007, p<.001\right]$. The lag effect reflected a standard $\mathrm{AB}$, with worse performance at lag $2(82.9 \%)$ than at lag $8(87.8 \%)$. The reason for the rather small magnitude of the blink is likely to be that a categorization task was used. We have used several versions of the $\mathrm{AB}$ task in our lab and have consistently observed smaller effect sizes in categorization than in identification tasks. In addition, the categorical separation between targets (numbers and symbols) and distractors (letters) likely made the task easier (see Juola, Botella, \& Palacios, 2004). The compatibility effect indicated that accuracy was lower when T2 and T3 were incompatible $(84.0 \%)$ or response compatible $(83.6 \%)$ than when they were fully compatible $(88.4 \%)$. Given that T3 was presented only after $\mathrm{T} 2$, whereas the $\mathrm{T} 3$ response preceded the response to $\mathrm{T} 2$, this effect seems to reflect interactions in memory. That is, maintaining $\mathrm{T} 2$ in working memory must have been easier if the same stimulus was processed again between $\mathrm{T} 2$ encoding and retrieval.

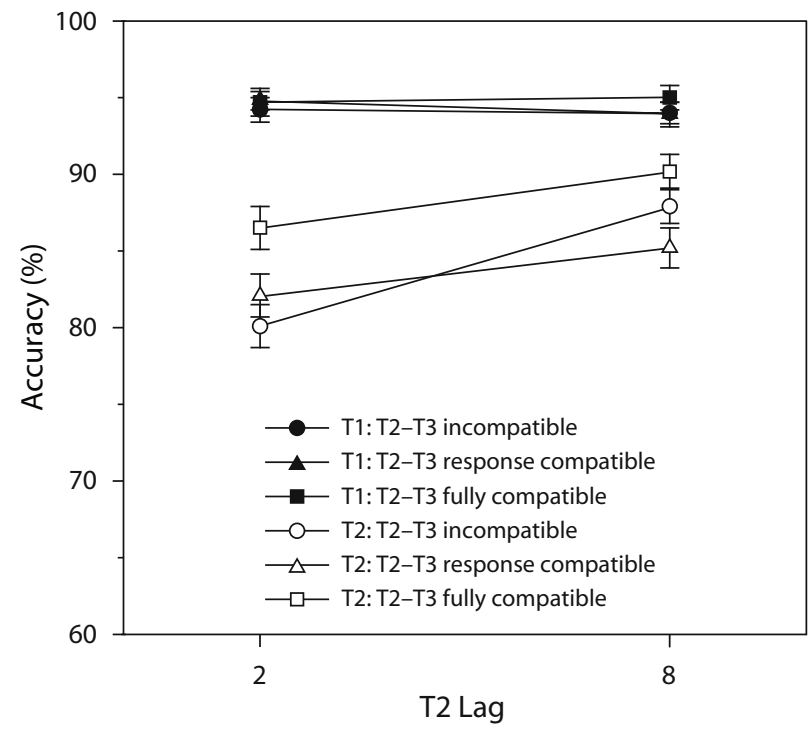

Figure 1. Target 1 (T1, black symbols) and conditional Target 2 (T2, white symbols) identification accuracy (percent correct) in Experiment 1, plotted separately for each compatibility relationship over T2 lag. Error bars in this and all other graphs represent standard errors of the means.
The interaction between lag and compatibility was also significant $\left[F(2,138)=10.41, M S_{\mathrm{e}}=.002, p<.001\right]$. As shown in Figure 1, the fully compatible and responsecompatible conditions do not change much across lag. Removing the incompatible condition rendered the interaction nonsignificant $(F<1)$, indicating that the other conditions did not differ from each other over lag. A separate test showed that performance at lag 8 in the incompatible condition even became slightly better than that in the response-compatible condition $\left[F(1,69)=7.06, M S_{\mathrm{e}}=.004, p<.01\right]$. This is an effect pattern that we will see again in Experiment 2 and that we will address in the General Discussion.

T3|T2. The left panel of Figure 2 shows conditional T3 accuracy as a function of lag. Accuracy was affected by compatibility $\left[F(1.6,109.3)=43.16, M S_{\mathrm{e}}=.012, p<\right.$ $.001]$, in that performance was worse when T2 and T3 were incompatible $(77.3 \%)$, better when they were response compatible $(85.1 \%)$, and best when they were fully compatible $(87.9 \%)$. This priming effect was not restricted to stimulus repetition, since the effects remained significant when the fully compatible condition was dropped from the analysis $\left[F(1,69)=37.0, M S_{\mathrm{e}}=.012, p<.001\right]$. That is, responding to $\mathrm{T} 3$ was better if it required the same response as T2, suggesting that perceiving T2 led to the activation of the associated response. This is in line with previous observations that merely registering a stimulus is sufficient to induce an immediate activation of stimulus-related response codes (e.g., Hommel, 1998; Hommel \& Eglau, 2002; Stoet \& Hommel, 1999). Lag did not affect T3 accuracy, presumably because T3 was very salient in appearance. The interaction between lag and compatibility was significant, however $\left[F(1.8,127.2)=11.04, M S_{\mathrm{e}}=.004, p<.001\right]$ : Whereas the fully compatible and the response-compatible conditions did not change much across lags, the incompatible condition showed a substantial improvement at lag 8 .

The right panel of Figure 2 displays T3 RTs. In contrast to the accuracy measure, T2 lag did have a large effect on the RTs $\left[F(1,69)=111.16, M S_{\mathrm{e}}=5,046.71, p<.001\right]$, indicating faster responses at the longer lag. This main effect should be interpreted with caution, since it was involved in an interaction discussed below. The compatibility effect was present as well $\left[F(1.8,122.9)=134.92, M S_{\mathrm{e}}=\right.$ 985.99, $p<.001]$ and followed the same pattern as in the accuracy analysis, showing faster responses in the fully compatible condition than in the response-compatible and the incompatible conditions. Although the overall difference between the incompatible and response-compatible conditions was modest, it was significant $[F(1,69)=4.66$, $\left.M S_{\mathrm{e}}=625.81, p<.05\right]$. Finally, the interaction between lag and compatibility was significant as well $[F(2,138)=$ $\left.8.24, M S_{\mathrm{e}}=448.09, p<.001\right]$. This occurred because, again, incompatibility of both the stimulus and response produced extra costs at lag 2 but not at lag 8; indeed, removing the incompatible condition from the analysis made the interaction disappear $(F<1)$. One factor that might have contributed to this pattern of results is a speed-accuracy trade-off that applied primarily to the incompatible condition. If this is so, the interaction effect might actually be somewhat underestimated, since the slope of the RT curve would be steeper if accuracy were held constant. ${ }^{1}$ 


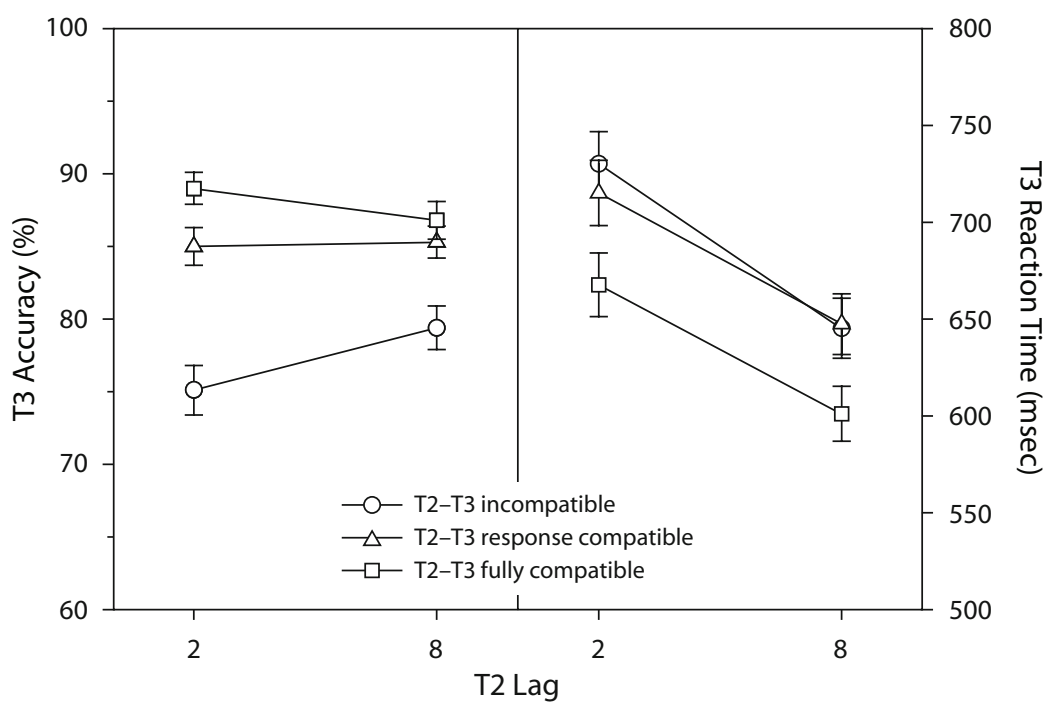

Figure 2. (Left) Target 3 (T3) identification accuracy (percent correct) in Experiment 1, given a correctly identified T2, plotted for each compatibility relationship over T2 lag. (Right) T3 reaction time (in milliseconds) plotted for the same conditions.

T3 after blinks. To test whether the reportability of a target (T2) would be of relevance for stimulus and response priming, we compared the effects of T2-T3 compatibility in trials in which $\mathrm{T} 2$ was missed or was successfully identified. To optimize statistical power, we averaged across the lag variable. Note that given the observed decrease of the difference between the response-compatible and the incompatible conditions with lag, this analysis underestimates the role of response priming, in particular for RTs. The ANOVA thus included the T2 blink variable (two levels: correct responses and errors) and the compatibility manipulation (three levels as before); see Figure 3 for T3 accuracy and RTs.

With T3 accuracy, T2 blink had a main effect: Missing $\mathrm{T} 2$ led to an average $\mathrm{T} 3$ identification of $75.7 \%$, whereas correct identification of T2 resulted in an improved 83.0\% accuracy $\left[F(1,69)=31.88, M S_{\mathrm{e}}=.018, p<.001\right]$. Compatibility remained significant in the present analysis $\left[F(2,138)=11.07, M S_{\mathrm{e}}=.011, p<.001\right]$. The incompatible condition showed the poorest performance, at $75.9 \%$, followed by the fully compatible condition at $80.6 \%$, and the response-compatible condition at $81.4 \%$; the difference between the latter two conditions was not reliable $(F<1)$.

The interaction between $\mathrm{T} 2$ blink and compatibility was significant, however $\left[F(2,138)=4.28, M S_{\mathrm{e}}=.012, p<\right.$ $.05]$. As shown in the figure, a blinked T2 changed the relationship between the compatibility conditions in two ways: First, the advantage of fully compatible over incompatible conditions was more pronounced if $\mathrm{T} 2$ could be

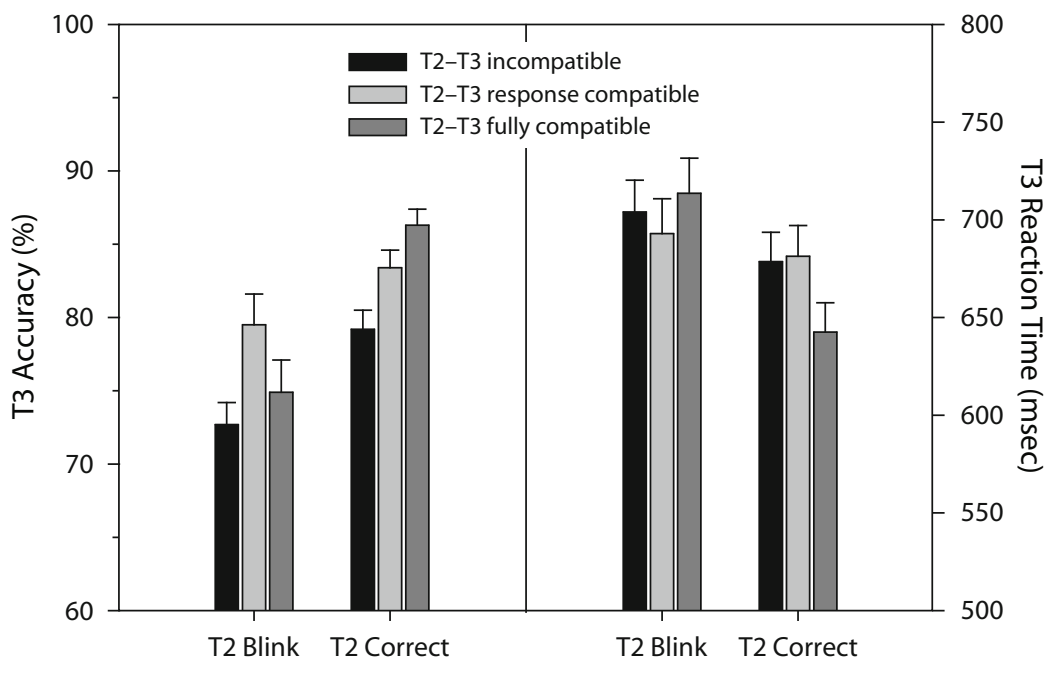

Figure 3. (Left) T3 identification accuracy (percent correct) in Experiment 1, for trials on which T2 was blinked and on which it was identified correctly. (Right) T3 reaction time (in milliseconds) plotted for the same conditions. 
reported than if it could not, and second, the advantage for response compatibility was greatly increased if $\mathrm{T} 2$ could not be reported. Closer examination confirmed this impression: Analyzing the differences with the blinked trials showed that performance in the response-compatible condition was better than that in the incompatible condition $\left[F(1,69)=8.14, M S_{\mathrm{e}}=.020, p<.01\right]$ and marginally better than in the fully compatible condition $[F(1,69)=$ $\left.3.62, M S_{\mathrm{e}}=.020, p<.06\right]$. In contrast, the analysis of the correctly identified trials showed higher performance in the fully compatible condition than in the responsecompatible condition $\left[F(1,69)=22.70, M S_{\mathrm{e}}=.003, p<\right.$ $.001]$, which in turn was still higher than performance in the incompatible condition $\left[F(1,69)=18.19, M S_{\mathrm{e}}=\right.$ $.002, p<.001]$. These observations suggest that the codes representing $\mathrm{T} 2$ are weaker in blinked than in correct trials, thereby reducing stimulus-specific priming. Response codes, however, do not seem to be affected by blinks, a pattern that will be seen again in Experiment 2 .

The analysis of T3 RTs showed a main effect of T2 blink: Responses were slower when T2 was missed $(704 \mathrm{msec})$ than when it was correctly identified $(667 \mathrm{msec})\left[F(1,69)=34.33, M S_{\mathrm{e}}=3,971.28, p<.01\right]$. Compatibility did not produce a main effect but interacted with blink $\left[F(1.8,122.2)=14.25, M S_{\mathrm{e}}=2,685.03, p<\right.$ $.01]$. The interaction was due to the particularly fast responses in the fully compatible condition when $\mathrm{T} 2$ could be reported; indeed, dropping the fully compatible condition rendered the interaction nonsignificant $(F<1.4)$.

It may be argued that T2 errors at lag 8 may not be due to the blink, and therefore confound the analysis. In order to control for that possibility, the same analyses were done on the data for lag 2 only. On T3 accuracy, the blink had the same main effect: If T2 was missed, accuracy for T3 averaged $75.1 \%$ correct, and correct identification of $\mathrm{T} 2$ resulted in $83.0 \%$ correct on $\mathrm{T} 3[F(1,69)=20.56$, $\left.M S_{\mathrm{e}}=.032, p<.001\right]$. Compatibility was much the same: When targets were incompatible, performance was $72.5 \%$; when they were fully compatible, it was $83.3 \%$; and when they were response compatible, it was $81.4 \%$ $\left[F(2,125)=16.79, M S_{\mathrm{e}}=.031, p<.001\right]$. Finally, the interaction between T2 blink and compatibility was significant as before $\left[F(2,114)=4.58, M S_{\mathrm{e}}=.037, p<\right.$ $.05]$. The means showed the exact same pattern as had the analysis including the lag 8 trials, and these means are summarized in Table 1. On T3 RTs, there was a main effect of the blink: As before, responses were slower when T2 was missed $(727 \mathrm{msec})$ than when it was correctly identified $(704 \mathrm{msec})\left[F(1,69)=7.14, M S_{\mathrm{e}}=7,741.7\right.$, $p<.01]$. Compatibility interacted in the same way with the blink $\left[F(2,107)=6.27, M S_{\mathrm{e}}=10,556.9, p<.01\right]$, and now also had a significant main effect $[F(1,102)=$ $\left.3.55, M S_{\mathrm{e}}=1,180.0, p<.05\right]$. Although RT was slightly higher overall, the pattern of means was again replicated, as shown in Table 2. These results showed that both approaches are highly similar and that no meaningful deviation occurs because of lag 8 errors potentially having a different source.

Taken together, our findings demonstrate stimulus and response priming in an $\mathrm{AB}$ task. However, they also show
Table 1

Mean T3 Identification Performance (\%) in Experiment 1, for Lag 2 Only

\begin{tabular}{cccc}
\hline & \multicolumn{3}{c}{ Compatibility } \\
\cline { 2 - 4 } T2 ID & None & Response & Stimulus \\
\hline Correct & 69.9 & 81.6 & 73.9 \\
Incorrect & 75.1 & 85.0 & 89.0 \\
\hline
\end{tabular}

that the two priming effects are sensitive to different conditions, and thus can be dissociated. Stimulus-specific priming is not (strongly) affected by T1-T2 lag, but it is highly sensitive to the strength of stimulus representation. Thus, on the one hand, T3 is reported faster and more accurately if it matches a previously presented stimulus, whether this stimulus was presented under high or low attentional demands (i.e., at a short or long lag). On the other hand, this benefit seems to vary with the success of the identification and, presumably, the consolidation of the prime, which eventually results in correct $\mathrm{T} 2$ report.

Response priming showed a different characteristic. It was instead immune to the blink (i.e., to T2 errors) and present even in blink trials, but it was affected by lag (i.e., the passage of time). At first sight, this might seem odd, especially given the small impact of lag on stimulus priming. However, let us assume that a short lag delays the processing of $\mathrm{T} 2$, whether that is because processing resources are still occupied with T1 consolidation (Chun \& Potter, 1995) or because T1 still monopolizes global communication in the brain (Gross et al., 2004). Given that the stimulus onset asynchrony (SOA) between T2 and T3 was short and fixed in Experiment 1, delaying the processing of T2 implies a shortening of the effective interval between T2 and T3 processing. At the same time, RTs to $\mathrm{T} 3$ were longer for lag 2 . Given that T3 accuracy was not impaired at lag 2, the RT effect seems to reflect a delay in response selection but not in stimulus processing. Hence, at lag 2, T2-induced processes took place closer to $\mathrm{T} 3$ processing and had more time to impact T3-related response selection than they did at lag 8 . To test whether this might have been the reason why response priming was stronger at the shorter lag, we manipulated the SOA between T2 and $\mathrm{T} 3$ in Experiment 2.

\section{EXPERIMENT 2}

The second experiment was identical to the first, with one exception. The lag between T1 and T2 was now kept constant at 8 , but the lag between $\mathrm{T} 2$ and $\mathrm{T} 3$ was varied between 2 and 6 instead. This change meant that target identification was relatively easy, because there was suf-

Table 2

Mean T3 Reaction Time (in Milliseconds) in Experiment 1, for Lag 2 Only

\begin{tabular}{cccc}
\hline & \multicolumn{3}{c}{ Compatibility } \\
\cline { 2 - 4 } T2 ID & None & Response & Stimulus \\
\hline Correct & 730 & 717 & 735 \\
Incorrect & 730 & 715 & 668 \\
\hline
\end{tabular}


ficient time available to process T2, and because T3 was a color oddball. At the same time, the temporal proximity between $\mathrm{T} 2$ and $\mathrm{T} 3$ processing was varied, which according to our hypothesis should affect response priming in ways similar to how T1-T2 lag did in Experiment 1.

\section{Method}

Participants. Thirty-eight new students participated for course credit or monetary compensation and met the same criteria as before. Two participants were excluded from analysis because they did not respond to the third target at all.

Apparatus, Stimuli, and Procedure. The experiment was constructed and run exactly like Experiment 1, with the noted change of the lag variable. Lag 6 was chosen rather than lag 8 to avoid having T3 appear too close to the end of the RSVP, which might ease its identification or consolidation in memory. The targets maintained their original appearance; that is, T1 (white) and T3 (red) were quite salient, whereas $\mathrm{T} 2$ was black like the distractors.

\section{Results and Discussion}

Repeated measures ANOVAs were carried out on accuracy on T1, accuracy on T2 $\mid \mathrm{T} 1$, and accuracy as well as RT on T3|T2. The independent variables were T2-T3 lag and T2-T3 compatibility.

T1. Figure 4 shows performance on $\mathrm{T} 1$ and on $\mathrm{T} 2$ given $\mathrm{T} 1$ for all conditions. T1 identification accuracy was not affected by any variable.

T2 | T1. Overall, accuracy was high, in line with expectations, and showed an effect of T3 lag: Performance increased from $86.9 \%$ at lag 2 to $88.8 \%$ at lag $6[F(1,35)=$ $\left.5.88, M S_{\mathrm{e}}=.003, p<.05\right]$. Compatibility had an effect as well: Performance rose from $83.8 \%$ in the incompatible and $86.6 \%$ in the response-compatible condition to $93.1 \%$ in the fully compatible condition $[F(1.2,41.0)=$ $\left.8.23, M S_{\mathrm{e}}=.034, p<.01\right]$. Here, the difference between incompatible and response compatible was not reliable $(F<1)$.

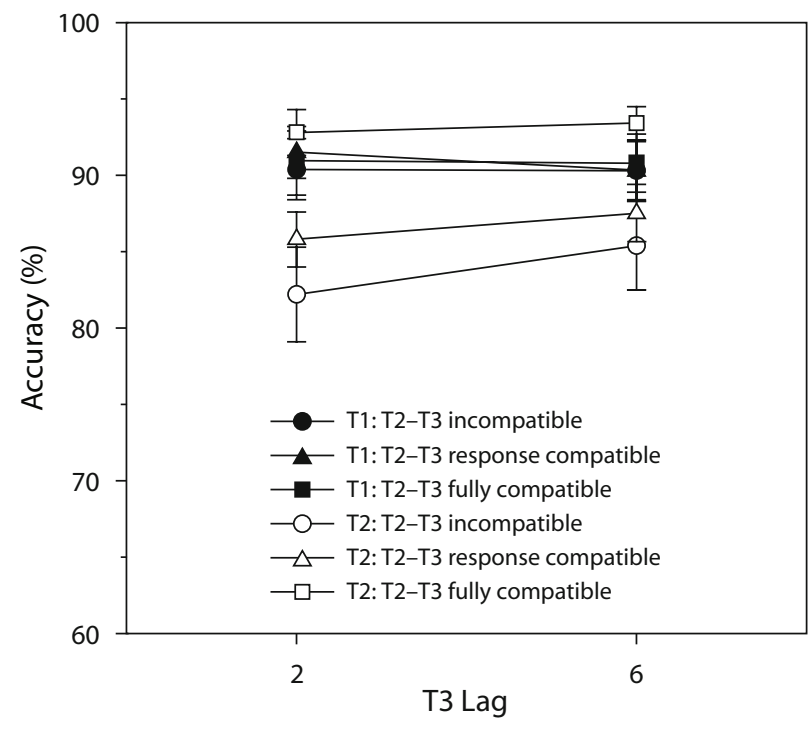

Figure 4.T1 (black symbols) and conditional T2 (white symbols) identification accuracy (percent correct) in Experiment 2, plotted separately for each compatibility relationship over $\mathrm{T} 3 \mathrm{lag}$.
T3 | T2. Figure 5 (left panel) shows T3 accuracy as a function of T2-T3 lag for each compatibility condition. Compatibility had a main effect: Accuracy was $83.1 \%$ when T2 and T3 were incompatible, compared with $83.9 \%$ when they were response compatible and $87.4 \%$ when fully compatible $\left[F(1.7,58.8)=5.23, M S_{\mathrm{e}}=.009, p<\right.$ $.05]$. The absence of an overall lag effect was as expected. Also expected was the interaction between lag and compatibility $\left[F(2,70)=11.64, M S_{\mathrm{e}}=.003, p<.001\right]$. As in Experiment 1, this effect was entirely due to the incompatible condition, since removing this condition made the interaction disappear $(F<1)$.

T3 RTs are plotted in the right panel of Figure 5. Again as predicted, the overall picture was virtually identical to that obtained in Experiment 1. The lag effect $[F(1,35)=$ 304.81, $\left.M S_{\mathrm{e}}=2,915.01, p<.001\right]$ showed again that responses were slower at the shorter lag. Since this effect was again involved in an interaction, however, this description should be interpreted with caution. Compatibility yielded a main effect $\left[F(1.5,51.4)=16.02, M S_{\mathrm{e}}=\right.$ $3,233.86, p<.001]$ and interacted with lag $[F(2,70)=$ $\left.6.68, M S_{\mathrm{e}}=896.50, p<.01\right]$. As in Experiment 1, this interaction was entirely due to the incompatible condition (i.e., removing this condition eliminated it), which had the poorest performance at lag 2 but not at lag 6 .

T3 after blinks. As in Experiment 1, an additional analysis was run to see how successful report of T2 affected the results. Again, the data were averaged over lag (which again implied an underestimation of response priming) and categorized according to whether T2 was blinked or reported correctly. Four participants had an insufficient number of observations in one or more cells of the design, because $\mathrm{T} 2$ errors were infrequent at the fixed T1-T2 lag of 8, and so were excluded from the present analysis. In general, the variance was higher than in Experiment 1 , since there were fewer participants. Figure 6 shows the means for T3 accuracy and RT as a function of T2 blink.

In general, the outcome pattern was almost a copy of that obtained in Experiment 1. T2 blink had an effect on T3 accuracy, in that performance decreased from $84.2 \%$ with correct report to $73.3 \%$ when $\mathrm{T} 2$ was missed $[F(1,31)=$ $\left.18.92, M S_{\mathrm{e}}=.030, p<.001\right]$. Compatibility continued to have an effect $\left[F(1.4,43.2)=5.41, M S_{\mathrm{e}}=.033, p<\right.$ $.05]$, indicating that performance was best for response compatibility, at $83.4 \%$, followed by full compatibility at $78.2 \%$, and finally incompatibility at $74.6 \%$. Finally, the interaction between T2 blink and compatibility was also significant $\left[F(1.5,47.3)=5.68, M S_{\mathrm{e}}=.033, p<\right.$ $.01]$. Whereas the response-compatible condition was immune to the occurrence of a blink and remained constant at $83.4 \%$, this was not the case for the incompatible and fully compatible conditions, both of which degraded when a blink occurred (from $82.3 \%$ to $66.9 \%$ and from $86.8 \%$ to $69.6 \%$, respectively). More specific analyses showed that performance in the response-compatible condition on blinked trials was higher than in the incompatible condition $\left[F(1,31)=27.45, M S_{\mathrm{e}}=.016, p<.001\right]$ and also than in the fully compatible condition $[F(1,31)=5.87$, $\left.M S_{\mathrm{e}}=.051, p<.05\right]$. In nonblink trials, performance 


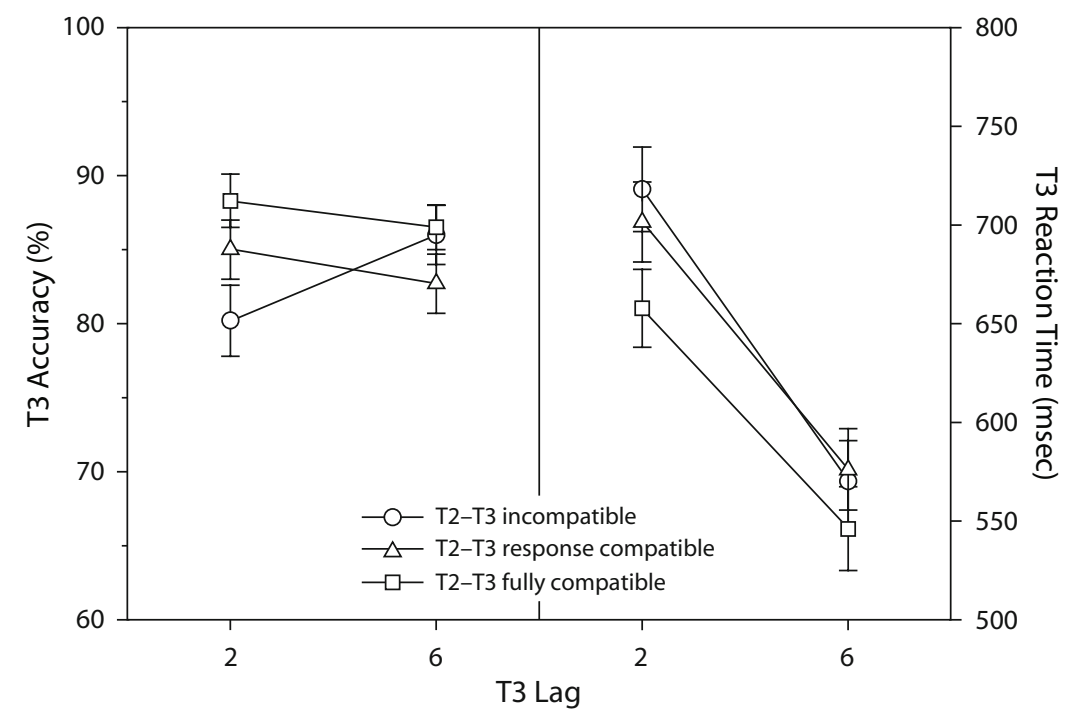

Figure 5. (Left) T3 identification accuracy (percent correct) in Experiment 2, given $\mathrm{T} 2$ correct, plotted for each compatibility relationship over $\mathrm{T} 3$ lag. (Right) $\mathrm{T} 3$ reaction time (in milliseconds) plotted for the same conditions.

was better in the fully compatible condition than in the response-compatible condition $\left[F(1,31)=7.75, M S_{\mathrm{e}}=\right.$ $.002, p<.01]$, but the latter and incompatible conditions did not differ $(F<1)$.

T3 RT was also affected by the presence of a blink $\left[F(1,31)=31.68, M S_{\mathrm{e}}=5,576.0, p<.001\right] . \mathrm{RT}$ was slower when T2 was missed $(684 \mathrm{msec})$ than when it was not $(624 \mathrm{msec})$. As observed previously in Experiment 1, compatibility did not have an effect on its own, but its interaction with $\mathrm{T} 2$ blink did $\left[F(2,62)=3.15, M S_{\mathrm{e}}=\right.$ $3,206.23, p<.05]$. The difference between the responseand fully compatible conditions was the only one that remained significant when taken separately $[F(1,31)=$ $\left.6.72, M S_{\mathrm{e}}=3,003.07, p<.05\right]$. The difference between the incompatible and response-compatible conditions came close $\left[F(1,31)=3.80, M S_{\mathrm{e}}=1,341.17, p<.06\right]$, but the last pair, incompatible versus fully compatible, did not $(F<1)$. Again the response-compatible condition was the most immune to the occurrence of an AB. Whereas response compatibility had a beneficial effect of $20 \mathrm{msec}$ when an error occurred, the effect of full compatibility was largest when T2 was identified correctly, at $30 \mathrm{msec}$.

As predicted, the manipulation of the lag between T2 and T3 has an effect comparable to the variation of T1-T2 lag in Experiment 1. Evidence of stimulus and response priming was again obtained, and again, response priming was more sensitive to the lag manipulation, whereas stimulus priming was more sensitive to the success or fail-

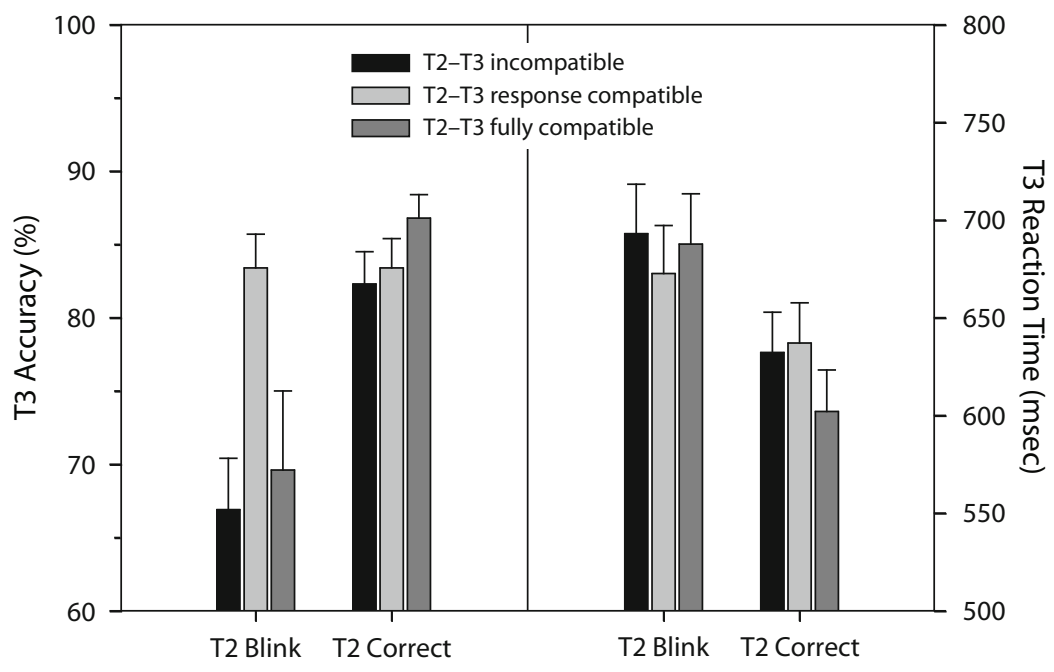

Figure 6. (Left) T3 identification accuracy (percent correct) in Experiment 2, for trials on which T2 was blinked and on which it was identified correctly. (Right) T3 reaction time (in milliseconds) plotted for the same conditions. 
ure to report $\mathrm{T} 2$. This confirms our expectation that processing T2 to some degree (which may or may not result in successful report) is sufficient to prime the associated response, but that this priming (or its impact) changes over time.

\section{GENERAL DISCUSSION}

Both experiments reported here showed a distinct pattern of priming for response- and stimulus-related compatibility between targets in an RSVP stream. In addition, effects that were more specific were due to the interplay between compatibility and intertarget lag and between compatibility and T2 blink. By itself, the presence of a facilitating effect could be considered surprising, given the opposite pattern (of repetition blindness) observed by Shapiro et al. (1997). The reason why the present results do not match those of Shapiro et al. might be that the present paradigm required only a target-matching response, whereas Shapiro et al. required full identification of their targets. One might also suspect that the temporal interval between targets was involved in some way; the present study employed varying target intervals and onsets, and this might have made it harder to strategically predict target onset. Furthermore, the targets used by Shapiro et al. did not differ from each other, nor from the distractors, which might have increased identity confusion. The combination of a predictable onset and confusable targets might have led to a different mode of detection. Finally, repetition blindness has been explained in terms of types (abstract categories) and tokens (specific instances) (Kanwisher, 1987). Whereas classic priming is due to a match in type between targets, repetition blindness is attributed to the increased difficulty of creating multiple tokens for a repeated type within a short time interval. In the present paradigm, the conditions for repetition blindness to occur were not met, and the conditions instead lent themselves more to classical priming. In the fully compatible condition, both the type information and token information were completely identical (as opposed to the difference in typeface used by Shapiro et al., for instance), and in the response-compatible condition neither type nor token matched.

In any case, the present experiments demonstrate that targets appearing in an RSVP stream not only activate their respective stimulus codes, but also the codes of associated responses, even under conditions in which the response is to be executed much later in time. This is true even when the stimulus itself cannot be reported, suggesting that stimulus-response translation proceeded automatically and independent of the attentional capacity limitations caused by the blink. At first sight, this may seem to be inconsistent with the frequent observation in dual-task studies that response selection draws on attentional resources to a degree that prohibits the concurrent selection of other responses (Pashler, 1994) or the consolidation of stimulus information in working memory (Jolicœur \& Dell'Acqua, 1998). However, capacity-free response priming has been found in dual-task studies as well (Hommel, 1998; Logan \& Schulkind, 2000), which suggests that response activation and response selection are different processes (Hommel, 1998; Jolicœur et al., 2002). If so, the present study can be taken to indicate automatic, stimulus-induced response activation, but not automatic response selection. Indeed, the main effects of lag on T3 RTs in both experiments suggest that T3-related response selection was delayed if the consolidation of $\mathrm{T} 2$ was not yet completed. In other words, stimulus consolidation weakened and delayed response selection but not response activation. This also provides a way in which the interaction between the $\mathrm{AB}$ and response selection found both by Jolicœur and colleagues (Jolicœur, 1998; Jolicœur \& Dell'Acqua, 1998) and in the present findings can be reconciled. In our paradigm, T3-related response selection was also delayed if $\mathrm{T} 2$ processing was not yet ready, but our measures of response activation (as evidenced by response priming) show that this activation itself was independent of actual selection - and as such would not interact with the blink.

Interestingly, we found evidence that stimulus and response priming are sensitive to different conditions. Response priming was more sensitive to the temporal proximity between the prime and the probed event (i.e., the primed response) than was stimulus priming, but it was less sensitive to the $\mathrm{AB}$. If we attribute stimulus and response priming to the activation of stimulus and response codes, respectively, this dissociation points to the relative independence of the two types of codes. That is, the response codes that were responsible for the priming effects were apparently more robust than, and not tightly bound to, the related target representations. This is particularly interesting in view of the observation that stimulus and response representations affect succeeding stimuli and responses in an interactive fashion (Hommel, 1998), suggesting that stimuli become integrated with the responses they accompany (Hommel, 2004). The fact that the present findings do not show similar evidence of stimulusresponse integration may thus be taken to imply that stimulus and response priming take place before that integration process. Indeed, recent studies support the idea that stimulus-response integration requires, and thus only follows, the execution of the response (Colzato, Warrens, \& Hommel, 2006; Hommel, 2005).

The benefits of response priming in the incompatible condition were reliable, but also decreased as the interval between prime and primed response increased. This was true for $\mathrm{T} 3$ performance in both experiments and for T2 performance in Experiment 1. One interpretation of this interaction is that stimulus presentation activates the code of the corresponding response but that this activation decays over time. Hence, response priming may only be transient. However, note that almost all of our graphs show almost perfectly parallel lines for the response-compatible and the fully compatible conditions, and all statistical results suggest that the incompatible condition is the true source of the interaction between compatibility and lag.

Oddly enough, in two cases (T2 accuracy in Experiment 1 and T3 accuracy in Experiment 2), the incompatible condition yielded even better performance than the response-compatible condition at the longer lag. Paired 
$t$ tests suggested these differences $[t(69)=2.7, p<.01$, and $t(35)=2.6, p<.05$, respectively], even though correction for multiple comparisons by means of Tukey tests indicated that they were not fully reliable $\left(t_{\text {crit }}=2.94\right.$ and $t_{\text {crit }}=3.01$, respectively). Still, to at least provide an account of this apparent trend, we will discuss its possible implications; namely, the observed pattern might reflect a response alternation effect. It is known that response repetitions facilitate performance at short interresponse intervals but impair performance at longer intervals (Bertelson, 1961; Soetens, Boer, \& Hueting, 1985). Although this facilitation is commonly explained by code priming (i.e., a gain from leftover activation), the bias toward response alternation at longer lags has been taken to indicate a kind of gambler's fallacy - that is, a strategic preference for the alternative response (Bertelson, 1961; Soetens et al., 1985). Now, assume that the same kind of preference biased response choices in trials in which our participants were uncertain about the correct response or in which they missed the target altogether. The alternation bias would artificially increase performance in the incompatible condition (since the alternative to the primed response would actually be the correct one) but decrease performance in the two other conditions (where the alternative to the primed response would be incorrect).

According to this interpretation, response code activation would not be any less stable over time than stimulus activation, but its impact on response selection might be outweighed by a bias toward response alternations at longer lags. Indeed, to make such an explanation viable, response activation should be particularly stable, which fits with the observation that response repetition effects are commonly more pronounced than stimulus repetition effects (Bertelson, 1965). This interpretation also fits with our finding that response priming was much more immune to the $\mathrm{AB}$ (i.e., to whether or not the prime was consciously perceived and could be reported) than was stimulus priming. This might even suggest that response code activation is more robust, or at least more inert, than stimulus code activation, but an alternative explanation is possible: Namely, the processes that are responsible for the $\mathrm{AB}$ may have a greater impact on stimulus codes than on response codes. For instance, consolidating a target may induce, or even require, the suppression of alternative target codes but not of response codes. If so, stimulus codes may not be more vulnerable by nature, but only suffer more from consolidation processes.

Figure 7 summarizes our assumptions made so far. Perceiving a target stimulus triggers stimulus coding processes - that is, the activation of codes representing the features of the stimulus. Activated stimulus codes are automatically "translated" into responses; that is, stimulus code activation spreads to associated response codes. Both of these coding processes are capacity unlimited in the present paradigm (as in other paradigms) and can be carried out in parallel (Hommel, 1998; Jolicœur et al., 2002). However, reporting a stimulus requires the consolidation of its codes into working memory, a capacity-limited process that may not be completed under high attentional demands (Jolicœur \& Dell’Acqua, 1998). Response selec-

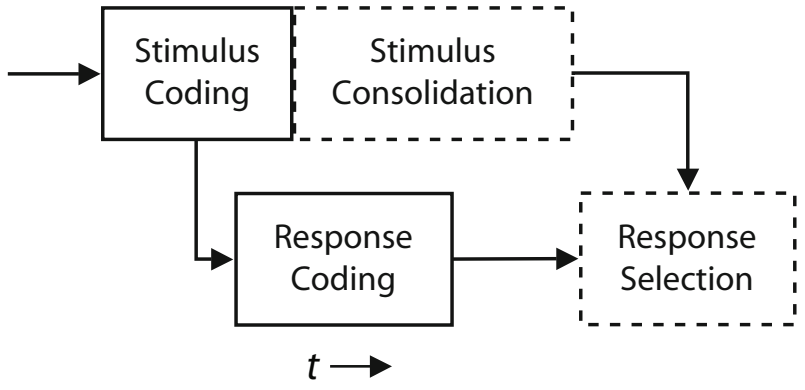

Figure 7. Process model of stimulus and response priming for the present dual-task paradigm. Stimulus and response coding are capacity-unlimited processes that can be carried out in parallel, whereas stimulus consolidation and response selection are capacity-limited processes that cannot overlap.

tion proper- that is, the process that integrates activated response codes into an executable action plan (Stoet \& Hommel, 1999) - is also capacity limited. Stimulus consolidation and response selection thus cannot overlap in time, which explains the lag main effects on RTs in the present study as well as the interactions between response selection and stimulus consolidation in the experiments of Jolicœur and Dell'Acqua. More generally speaking, our findings suggest that what keeps our conscious experience busy is not so much the processing of events or the induction of action tendencies, but instead what we want to do with the events and which tendencies we want to have win out.

\section{AUTHOR NOTE}

E.G.A. is now at Ludwig Maximilian University, Munich. Correspondence relating to this article may be sent to E. G. Akyürek, Ludwig Maximilian University, Department of Psychology, Experimental Psychology, Leopoldstraße 13, 80802 Munich, Germany (e-mail: akyurek@psy.lmu.de).

\section{REFERENCES}

Bertelson, P. (1961). Sequential redundancy and speed in a serial twochoice responding task. Quarterly Journal of Experimental Psychology, 13, 90-102.

Bertelson, P. (1965). Serial choice reaction-time as a function of response versus signal-and-response repetition. Nature, 206, 217-218.

Broadbent, D. E., \& Broadbent, M. H. P. (1987). From detection to identification: Response to multiple targets in rapid serial visual presentation. Perception \& Psychophysics, 42, 105-113.

Chun, M. M., \& PotTer, M. C. (1995). A two-stage model for multiple target detection in rapid serial visual presentation. Journal of Experimental Psychology: Human Perception \& Performance, 21, 109-127.

Colzato, L. S., Warrens, M. J., \& Hommel, B. (2006). Priming and binding in and across perception and action: A correlational analysis of the internal structure of event files. Quarterly Journal of Experimental Psychology, 59, 1785-1804.

Eimer, M., \& SCHLAGHECKEN, F. (1998). Effects of masked stimuli on motor activation: Behavioral and electrophysiological evidence. Journal of Experimental Psychology: Human Perception \& Performance, 24, 1737-1747.

Gross, J., Schmitz, F., Schnitzler, I., Kessler, K., Shapiro, K., HomMEL, B., \& SCHNITZLER, A. (2004). Modulation of long-range neural synchrony reflects temporal limitations of visual attention in humans. Proceedings of the National Academy of Sciences, 101, 13050-13055.

Hommel, B. (1998). Automatic stimulus-response translation in dualtask performance. Journal of Experimental Psychology: Human Perception \& Performance, 24, 1368-1384. 
Hommel, B. (2004). Coloring an action: Intending to produce color events eliminates the Stroop effect. Psychological Research, 68, 74-90.

Hommel, B. (2005). How much attention does an event file need? Journal of Experimental Psychology: Human Perception \& Performance, 31, 1067-1082.

Hommel, B., \& Eglau, B. (2002). Control of stimulus-response translation in dual-task performance. Psychological Research, 66, 260-273.

JOLICCEUR, P. (1998). Modulation of the attentional blink by on-line response selection: Evidence from speeded and unspeeded Task $\mathrm{K}_{1}$ decisions. Memory \& Cognition, 26, 1014-1032.

Joliceeur, P., \& Dell'ACQuA, R. (1998). The demonstration of shortterm consolidation. Cognitive Psychology, 36, 138-202.

Jolicceur, P., Tombu, M., Oriet, C., \& Stevanovski, B. (2002). From perception to action: Making the connection. In W. Prinz \& B. Hommel (Eds.), Common mechanisms in perception and action: Attention and performance XIX (pp. 558-586). Oxford: Oxford University Press.

Juola, J. F., Botella, J., \& Palacios, A. (2004). Task- and locationswitching effects on visual attention. Perception \& Psychophysics, 66, 1303-1317.

KANWISHER, N. G. (1987). Repetition blindness: Type recognition without token individuation. Cognition, 27, 117-143.

Leuthold, H., \& Kopp, B. (1998). Mechanisms of priming by masked stimuli: Inferences from event-related brain potentials. Psychological Science, 9, 263-269.

LoACH, D., \& Marí-Beffa, P. (2003). Post-target inhibition: A temporal binding mechanism? Visual Cognition, 10, 513-526.

Logan, G. D., \& Schulkind, M. D. (2000). Parallel memory retrieval in dual-task situations: I. Semantic memory. Journal of Experimental Psychology: Human Perception \& Performance, 26, 1072-1090.

Luck, S. J., Vogel, E. K., \& Shapiro, K. L. (1996). Word meanings can be accessed but not reported during the attentional blink. Nature, 383, 616-618.

Martens, S., Wolters, G., \& van RaAmsdonk, M. (2002). Blinks of the mind: Memory effects of attentional processes. Journal of Experimental Psychology: Human Perception \& Performance, 28, 1275-1287.
Neumann, O., \& Klotz, W. (1994). Motor responses to non-reportable, masked stimuli: Where is the limit of direct parameter specification? In C. Umiltà \& M. Moscovitch (Eds.), Attention and performance $X V$ : Conscious and nonconscious information processing (pp. 123-150). Cambridge, MA: MIT Press, Bradford Books.

PAshler, H. (1994). Dual-task interference in simple tasks: Data and theory. Psychological Bulletin, 116, 220-244.

Raymond, J. E., Shapiro, K. L., \& ARnell, K. M. (1992). Temporary suppression of visual processing in an RSVP task: An attentional blink? Journal of Experimental Psychology: Human Perception \& Performance, 18, 849-860.

Schneider, W., \& Shiffrin, R. M. (1977). Controlled and automatic human information processing: I. Detection, search, and attention. Psychological Review, 84, 1-66.

Shapiro, K. L., Driver, J., Ward, R., \& Sorensen, R. E. (1997). Priming from the attentional blink: A failure to extract visual tokens but not visual types. Psychological Science, 8, 95-100.

Soetens, E., Boer, L. C., \& Hueting, J. E. (1985). Expectancy or automatic facilitation? Separating sequential effects in two-choice reaction time. Journal of Experimental Psychology: Human Perception \& Performance, 11, 598-616.

Stoet, G., \& Hommel, B. (1999). Action planning and the temporal binding of response codes. Journal of Experimental Psychology: Human Perception \& Performance, 25, 1625-1640.

Vogel, E. K., Luck, S. J., \& ShapIRo, K. L. (1998). Electrophysiological evidence for a postperceptual locus of suppression during the attentional blink. Journal of Experimental Psychology: Human Perception \& Performance, 24, 1656-1674.

\section{NOTE}

1. We thank an anonymous reviewer for suggesting this interpretation.

(Manuscript received April 21, 2006; revision accepted for publication March 14, 2007.) 